\title{
A Novel "Subset Splitting" Procedure for Digital Image Correlation on Discontinuous Displacement Fields
}

\author{
J. Poissant • F. Barthelat
}

Received: 7 August 2008 / Accepted: 5 January 2009 / Published online: 3 February 2009

(C) Society for Experimental Mechanics 2009

\begin{abstract}
Digital Image Correlation (DIC) is an easy to use yet powerful approach to measure displacement and strain fields. While the method is robust and accurate for a variety of applications, standard DIC returns large error and poor correlation quality near displacement discontinuities such as cracks or shear bands. This occurs because the subsets used for correlation can only capture continuous deformations from the reference to the deformed image. As a result the regions around discontinuities are typically removed from the area of interest, before or after analysis. Here, a novel approach is proposed which enables the subset to split in two sections when a discontinuity is detected. This method enables the measurement of "displacement jumps", and also of displacements and strains right by the discontinuity (for example a crack profile or residual strains in the wake). The method is validated on digitally created images based on mode I and mode II asymptotic displacement fields, for both sub-pixel and super-pixel crack opening displacements. Finally, an actual fracture experiment on a high density polyethylene (HDPE) specimen demonstrates the robustness of the method on actual images. Compared to other methods capable of handling discontinuities, this
\end{abstract}

\footnotetext{
J. Poissant

Department of Mechanical Engineering,

MacDonald Engineering Building, Room 53, McGill University,

817 Sherbrooke Street West,

Montreal, Quebec H3A 2K6, Canada

e-mail: jeffrey.poissant@mail.mcgill.ca

F. Barthelat ( $\square$, SEM Member)

Department of Mechanical Engineering,

MacDonald Engineering Building, Room 351, McGill University,

817 Sherbrooke Street West,

Montreal, Quebec H3A 2K6, Canada

e-mail: francois.barthelat@mcgill.ca
}

novel "subset-splitting" procedure offers the advantage of being a direct extension of the now popular standard DIC, and can therefore be implemented as an "upgrade" to that method.

Keywords Digital image correlation and subset splitting . Discontinuous displacement fields · Novel DIC technique validation - Experimental fracture mechanics . Crack opening displacement and crack profile

\section{Introduction}

Digital Image Correlation (DIC), since its introduction about 25 years ago [1], has become a popular method for the measurement of displacement and strain fields. DICbased mechanical experiments are generally easier to setup and to carry out than experiments involving other strain measurement methods such as strain gages or speckle interferometry. DIC is also a very flexible technique that can be applied to any type of digital image (photography, optical, electron or atomic force microscopy). The method correlates the intensity distribution of small subsets of pixels from a reference image and a deformed image to determine the full displacement fields [1, 2]. Standard DIC is an accurate and robust method in a large number of applications, but the method fails when strong displacement discontinuities such as cracks [3, 4] or shear bands [5] are present in the image. More specifically, the subset is unable to capture displacement jumps; the measured displacements are erroneous in a region centered on the discontinuity and of width equal to the subset size used for the analysis. It is therefore common to either not correlate the image subsets near discontinuities, or to correlate them and remove the erroneous values afterwards [6-9]. These areas can actually 
be removed automatically from the final results by discarding subsets with insufficient correlation quality (i.e. whose correlation coefficient is below or above a set value, depending on which definition is used for the correlation coefficient) [10]. While these approaches facilitate data analysis, the displacements and strains near a crack or shear band can still not be determined with standard DIC. This becomes a limitation for cases where one wants to measure the residual strains in the wake of a crack or to compute $J$ integrals on closed contours around the crack tip.

Alternate correlation methods that overcome this limitation have been recently developed. For example, Jin and Bruck developed a pointwise DIC method, which optimizes the in-plane displacements of all the pixels within a subset simultaneously, thereby enabling the measurement of discontinuous displacements [11]. This technique requires genetic algorithms because of the large number of unknowns that are optimized at one time. Réthoré, Hild, and Roux developed another approach called extended $D I C$, which is capable of handling cracks $[12,13]$. The method is based on the finite element method: the image is meshed with elements, and the global correlation between reference and deformed image is minimized to yield the nodal displacements. This "global" approach differs fundamentally from standard digital image correlation, where subsets of the image are correlated independently from each other. The extended DIC then borrows from the extended finite element method (XFEM) [14] by "enriching" the shape functions of the elements to take into account the presence of a crack. While pointwise DIC can provide good results in the presence of a crack, this technique is computationally expensive. In addition, both pointwise and extended DIC differ fundamentally from standard DIC. Here we propose a third route to handling displacement discontinuities, which is a direct extension of the subset-based standard DIC and which in this sense can be viewed as an "add-on" to that method. While the core of the method remains the same, the subset is allowed to split in two sections when a discontinuity is detected. The theory and implementation of subset splitting are presented in the first section of this article. Three examples follow: two validations on digitally generated images of cracked samples in mode I and mode II, and a qualitative application from an actual fracture test.

\section{Digital Image Correlation and Subset Splitting}

a. Standard method:

The subset splitting procedure is a direct extension of the standard two dimensional DIC, which is briefly summarized in this section (more details can be found elsewhere [15-18]). For standard DIC, digitized images of the randomly speckled specimen surface are acquired (by a CCD camera for example) in the undeformed state (reference image, with intensity distribution $f(X, Y)$ ) and in the deformed state (deformed image, with intensity distribution $g(X, Y)$ ). The deformed image is interpolated to obtain a continuous description of the intensity distribution, (fifth order B-splines were found to yield the best results [17]). The displacement of a pixel located at $\left(X_{\mathrm{p}}, Y_{\mathrm{p}}\right)$ in the reference image is determined using subset shape functions to map the displacement of a square subset centered on that pixel [Fig. 1(a)]. Linear subset shape functions were chosen for the present work:

$$
u_{\text {subset }}(i, j)=u+\frac{\partial u}{\partial x} i+\frac{\partial u}{\partial y} j \quad v_{\text {subset }}(i, j)=v+\frac{\partial v}{\partial y} j+\frac{\partial v}{\partial x} i
$$

Where $u$ and $v$ are the center of the subset's displacements and $\frac{\partial u}{\partial x}, \frac{\partial u}{\partial y}, \frac{\partial v}{\partial y}, \frac{\partial v}{\partial x}$ are the linear subset deformations [Fig. 1(a)]. The best match for the parameters, $\vec{q}=\left(u, v, \frac{\partial u}{\partial x}, \frac{\partial u}{\partial y}, \frac{\partial v}{\partial y}, \frac{\partial v}{\partial x}\right)$, maximizes the cross-correlation coefficient [15] or minimizes the least-squares correlation coefficient [16]. The least-squares correlation coefficient $C$ was selected for the present work to take advantage of the
Fig. 1 (a) Standard DIC: transformation of a reference subset into a deformed subset (adapted from Chu et al. [2]). (b) DIC with subset splitting: The deformed subset is split in two to accommodate for a displacement discontinuity

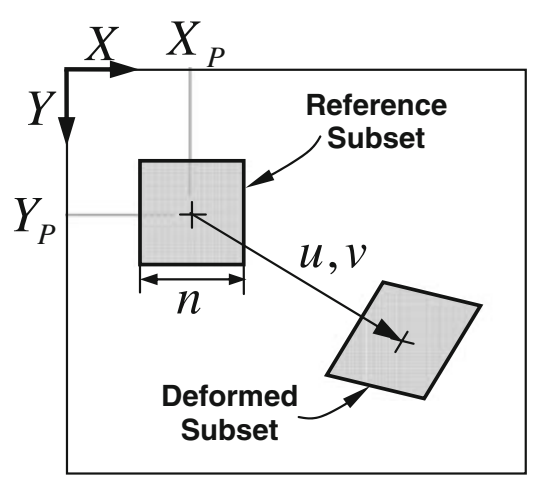

(a)

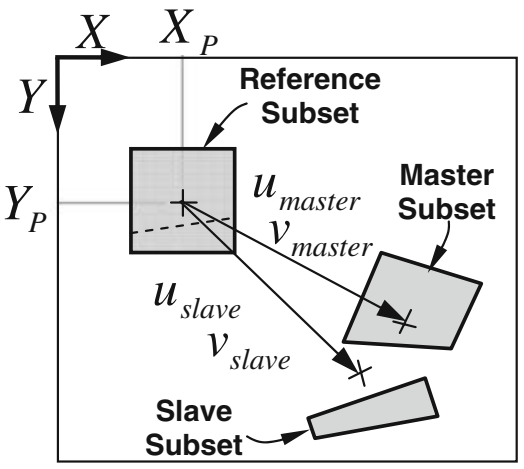

(b) 
Hessian approximation presented by Vendroux and Knauss

[16]:

$C(\overrightarrow{\mathbf{q}})=\frac{\sum_{j=-m}^{m} \sum_{i=-m}^{m}\left[\left\{f\left(X_{P}+i, Y_{P}+j\right)-g\left(X_{P}+i+u_{\text {subset }}(i, j), Y_{P}+j+v_{\text {subset }}(i, j)\right)\right\}^{2}\right]}{\sum_{j=-m}^{m} \sum_{i=-m}^{m}\left[f\left(X_{P}+i, Y_{P}+j\right)^{2}\right]}$

where $m=\frac{n-1}{2}$ and $n$ is the subset size. In equation (2) $C=0$ means a perfect match between reference and deformed subsets. To minimize $C$, a Newton-Raphson approach was implemented to find the zeros of the gradient of $C$ [15]. Thus, the optimized shape function parameters can be determined iteratively using the following formula, until a stopping condition is met:

$$
\overrightarrow{\mathbf{q}}_{i+1}-\overrightarrow{\mathbf{q}}_{i}=\Delta \overrightarrow{\mathbf{q}}_{i}=[\mathbf{H}]^{-1}(-\nabla \mathbf{C})
$$

Where $[\mathbf{H}]$ is the Hessian matrix and $\nabla C$ is the gradient of $C(\overrightarrow{\mathbf{q}})$. This iterative procedure can then be performed on several subsets thereby generating the full displacement field of the surface.

While this method is robust and accurate for a large number of applications, it fails near cracks or shear bands, because the subset shape function is unable to capture the strong displacement discontinuities associated with these features. This has been limiting the applications of DIC in fracture mechanics, and a common approach had been to simply not correlate pixels whose subset cross the discontinuity.

b. The subset splitting method

The subset splitting method is a direct extension of standard DIC. It enables the subset to split in two sections when a discontinuity is detected [Fig. 1(b)]. The approach is similar to "enriching" the subset shape functions with a discontinuous displacement field as in extended finite elements [14] or extended digital image correlation [12, 13]. Consider an image subset intersected by a displacement discontinuity (such as a crack or a shear band). The subset is assumed to be small enough so that the intersection can be modeled as a line with equation $j=a i+b$, where $(i, j)$ are subset coordinates (with origin at the subset center). Higher order polynomials could possibly be implemented to account for a non-linear shaped discontinuity, but the present work will consider only lines: Assuming that the subset is small with respect to the discontinuity's curvature, a line can model the discontinuity correctly. The line splits the subset into a "master section" which contains the subset center and the most pixels, and the "slave section" (Fig. 2). The pixels which are intersected by the line are excluded from the correlation coefficient calculations. The special cases where the line crosses the center pixel are automatically excluded from the analysis by the algorithm. These cases represent attempts to determine the displacement of pixels which lie on the discontinuity, which cannot be resolved. In other words, the crack has "cut" the pixel of interest in two; this pixel can arguably be on either crack face. After the subset is split, the pixels on either side of the splitting line are no longer expected to contain discontinuities and are assigned their own distinct sets of continuous, linear shape function parameters (i.e. $\overrightarrow{\mathbf{q}}_{\text {Master }}$ and $\overrightarrow{\mathbf{q}}_{\text {Slave }}$ ). However, the two sections each contain a fraction of the complete subset and therefore, both contribute to the same correlation coefficient $C$ (equation 2). In total, 14 parameters must be optimized in the subset splitting method: Two splitting line parameters (slope $a$ and $y$-intercept $b$ ), six mapping parameters for the master section and six mapping parameters for the slave section. In implementing the optimization it rapidly became evident that the splitting line parameters $a$ and $b$ had to be treated separately. Incorporating these line parameters into the Newton-Raphson method proved impractical because the partial derivatives with respect to $a$ and $b$ cannot be passed into the sums of $C$. Also, numerical derivatives proved troublesome as $C(\ldots, a, b)$ is not well behaved. Thus, the line parameters were not optimized directly. Instead, the optimized deformation parameters were assumed to yield optimized line parameters. This assumption is acceptable because the splitting line simply removes all pixels through which it crosses (Fig. 2). For example, let a horizontal discontinuity cross the subset at 0.25 pixels above the subset

Fig. 2 In this example a $9 \times 9$ subset is split by a discontinuity into a master section and a slave section. The grayed pixels which are intersected by the line are discarded from the correlation coefficient calculations 
center. The optimized line parameters would be $a=0, b=0.25$. However, using $a=0.0005, b=0.1$ would have exactly the same effect, since the same pixels would be removed. Specific details about computing the line parameters are outlined in the upcoming section. While the parameters of interest are only the displacement of the center pixel $u$ and $v$, which is contained by the master subset, there is still a need to track and optimize the translation and deformation of the slave section. This slave section is needed to add stability when determining the location of the splitting line. A "subset cropping" approach where the pixels from the slave section were discarded from the calculation of $C$ was unsuccessful and did not converge properly, because in all cases this approach encouraged the Master section to be as small as possible to minimize $C$.

\section{c. Subset Splitting Implementation}

The standard DIC and the subset splitting method were implemented in Matlab (The MathWorks, Natick, MA, USA). The flowchart for the algorithm is shown on Fig. 3. The first step is a standard DIC analysis on the entire area of interest. This first analysis typically yields acceptable results everywhere within the area of interest except near discontinuities such as cracks or shear bands, where the quality of the correlation is significantly lower. In a second step, the subsets whose correlation quality is poorer than a preset value (the "splitting threshold" $C_{\text {st }}$ ) are selected to be re-correlated with the subset splitting method. The optimization of the 14 split subset parameters requires initial guesses. For the first subset to undergo subset splitting, the
Fig. 3 Subset splitting algorithm flow chart

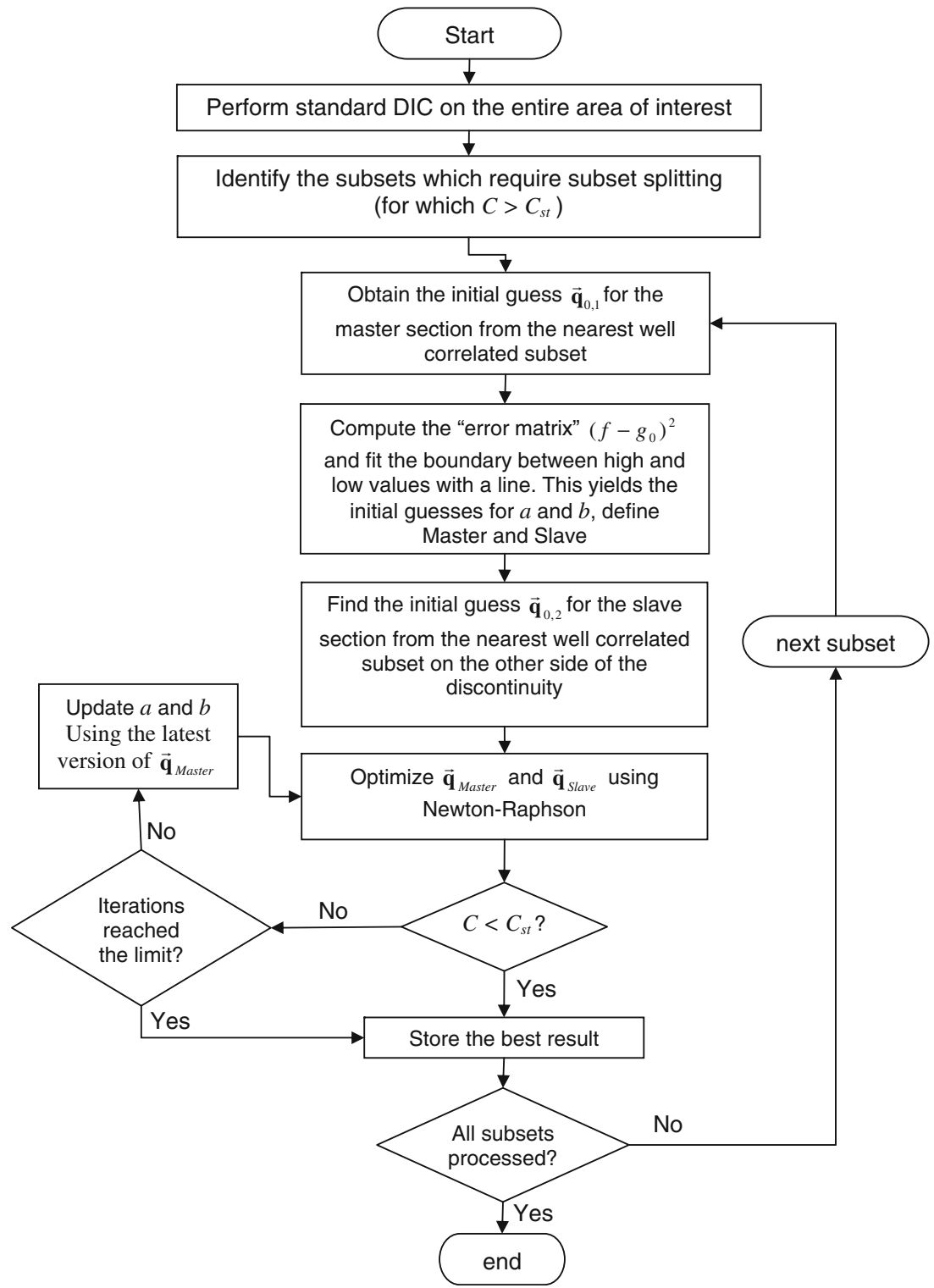


initial guess for the mapping of the master section is provided by the nearest subset with a valid $C$ (i.e., $C<C_{\mathrm{st}}$ ). With this initial guess $\overrightarrow{\mathbf{q}}_{0,1}$, a deformed subset $g_{0}(i, j)$ can be obtained, and compared with the entire reference subset $f(i, j)$. Computing $c(i, j)=\left(f(i, j)-g_{0}(i, j)\right)^{2}$ for each pixel of the subset yields a matrix which contains one section that is well correlated, and another section that is poorly correlated (high error values) as a consequence of the discontinuity. A straight line is then fitted onto the boundary between high and low $c(i, j)$ 's to yield an initial guess for $a$ and $b$. In order to find the first guess for the mapping of the slave section, the nearest subset with a valid $C$, (i.e. $C<C_{\mathrm{st}}$ ) on the opposite side of the discontinuity and normal to the splitting line, is located and its mapping stored as the initial guess for the slave section $\overrightarrow{\mathbf{q}}_{0,2}$. With these initial guesses, the mapping of the master and slave sections is then optimized with Newton-Raphson. In this process two gradients and Hessians are computed, one for each section. The gradient and Hessian of each section take into account only the pixels contained within that section, unlike $C$ which is composed of contributions from both sections. With these values, the deformation parameters can be optimized:

$$
\left\{\begin{array}{l}
\Delta \overrightarrow{\mathbf{q}}_{i \text { Master }}=\left\{[\mathbf{H}]^{-1}(-\nabla \mathbf{C})\right\}_{\text {Master }} \\
\Delta \overrightarrow{\mathbf{q}}_{i \text { Slave }}=\left\{[\mathbf{H}]^{-1}(-\nabla \mathbf{C})\right\}_{\text {Slave }}
\end{array}\right.
$$

For every update to $\overrightarrow{\mathbf{q}}_{\text {Master }}$ and $\overrightarrow{\mathbf{q}}_{\text {Slave }}$, a single correlation coefficient is obtained by piecing together each section's contribution. After $\overrightarrow{\mathbf{q}}_{\text {Master }}$ and $\overrightarrow{\mathbf{q}}_{\text {Slave }}$ have converged, if $C$ is lower than the splitting threshold $C_{\text {st }}$, then the subset is well correlated and the mapping parameters of the master subset are stored. If $C$ is still higher than $C_{\mathrm{st}}$, then the algorithm returns to update the splitting line parameters $a$ and $b$. To update $a$ and $b$, the latest $\overrightarrow{\mathbf{q}}_{\text {Master }}$ computed is used to obtain another $g_{0}(i, j)$, generate another error matrix $c(i, j)=\left(f(i, j)-g_{0}(i, j)\right)^{2}$, and using the same technique, update the splitting line. This procedure is repeated until the splitting tolerance is met, or until a specified number of iterations have exhausted the search for the best-fit. For the present work, the maximum number of iterations was set at five. Most subsets in the image, including those being split, converged within five iterations. Those that did not converge within that limit were subsets for which the slave section had only a few pixels, which were insufficient to achieve the threshold set for convergence. All of those subsets, however, achieved convergence for the master section. The maximum number of iteration was set to five by trial and error; it was found that increasing the number of maximum iterations allowed did not change the resulting displacement field.

\section{Validation and Application of Subset Splitting}

\section{Example 1: Digitally Created Horizontal Mode I Crack}

The subset splitting method was validated on a pair of images which were digitally created from a known displacement field. Note that no artificial noise was introduced into the images; therefore the results from Example 1 and 2 represent upper bounds on performance. For this first example a $500 \times 500$ pixel reference image of a random pattern was deformed according to a mode I asymptotic displacement field; a horizontal crack with the tip located at the center of the image:

$$
\begin{aligned}
& U(x, y)=\frac{K_{I}}{2 E} \sqrt{\frac{r}{2 \pi}}(1+v)[(2 \kappa-1) \cos (\theta / 2)-\cos (3 \theta / 2)] \\
& V(x, y)=\frac{K_{I}}{2 E} \sqrt{\frac{r}{2 \pi}}(1+v)[(2 \kappa+1) \sin (\theta / 2)-\sin (3 \theta / 2)]
\end{aligned}
$$

Here $(x, y)$ and $(r, \theta)$ are the Cartesian and polar coordinates centered on the crack tip, $K_{I}$ is the stress intensity factor, $E$ and $v$ are Young's modulus and Poisson's ratio, and $\kappa=(3-4 v)$ for plane strain. The Young's modulus and Poisson's Ratio were fixed at $70 \mathrm{GPa}$ and 0.3 respectively. The stress intensity factor was set to $1.5 \mathrm{GPa} \sqrt{\text { pixels }}$ to obtain a maximum crack opening displacement of about \pm 5 pixels. Another deformed image was generated using $K_{I}=300 \mathrm{MPa} \sqrt{\text { pixels }}$ in order to obtain a maximum crack opening displacement of about \pm 1 pixel. To generate each deformed image, two grids of size $500 \times 500$, representing the $x$ positions and y positions were created with the origin at the center. Using the displacement fields in equation (5), two more grids of equal size were generated to represent $U$ and $V$. The $U$ grid was added to the $x$ positions and the $V$ grid to the y positions to yield the deformed positions of the original grids. The intensity values of the reference image were then assigned to these deformed positions. The deformed image's intensity values were then calculated by interpolating the deformed reference intensities at the original grid positions. To mimic the captors of a CCD camera, the interpolated surface was sampled using a square grid around each pixel of the deformed image. Figure 4 shows the reference and deformed images used for this example, together with the area of interest (the crack is composed of interpolated intensities, in some cases hard to distinguish. Their location was circled on the figure). The displacement fields and correlation coefficients for standard DIC and subset splitting DIC are shown in Fig. 5, for super-pixel crack opening. For these analyses the subset size was 25 pixels (following Pan et al, [19]) and subsets were correlated every 2 pixels to ensure that almost every point along the faces of the discontinuities was processed by subset 
Fig. 4 Images used for the evaluation of the subset splitting method with a horizontal mode I crack: (a) Reference image and area of interest; (b) Super-pixel deformed image $(\mathrm{COD} \pm 5$ pixels); (c) Sub-pixel deformed image (COD \pm 1 pixels). For both deformed images the crack tip is in the center of the image

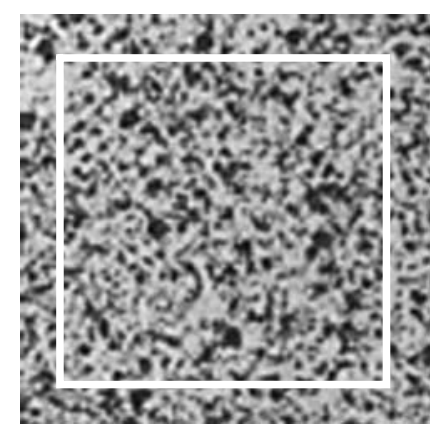

(a)

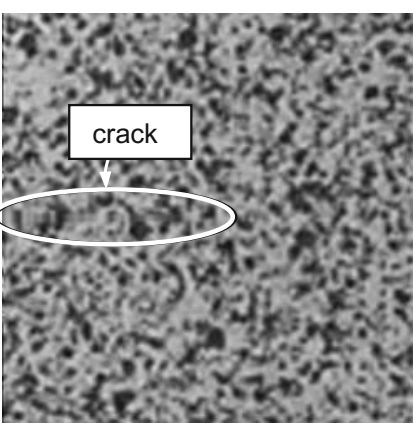

(b)

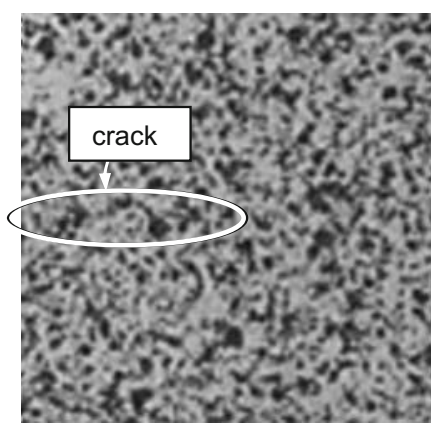

(c)

Fig. 5 Image correlation results from the mode I horizontal crack with super-pixel crack openings using standard DIC and DIC with subset splitting (all axes' units in pixels)

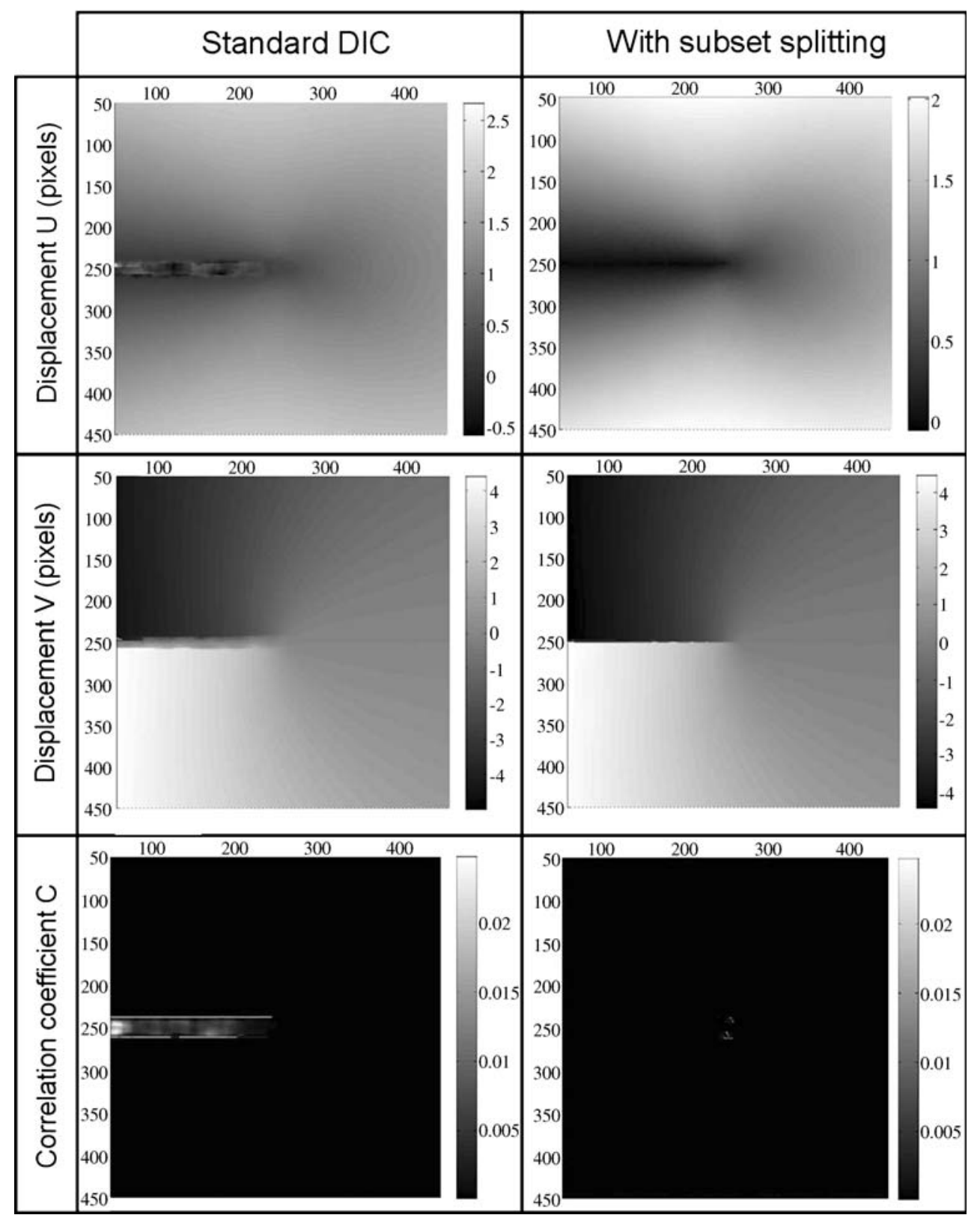


splitting; however, this is not a necessary condition for the subset splitting method (a coarser grid would not change the displacements computed by each subset). The preliminary run with standard DIC yielded correlation coefficients on the order of $10^{-6}$ far from the crack (i.e. good quality correlation) and on the order of $10^{-4}$ to $10^{-3}$ near the crack (i.e. poor correlation). Based on these values, a splitting tolerance of $10^{-5}$ was chosen by the user: Every subset with a correlation coefficient higher than $C_{\mathrm{st}}=10^{-5}$ underwent subset splitting. This
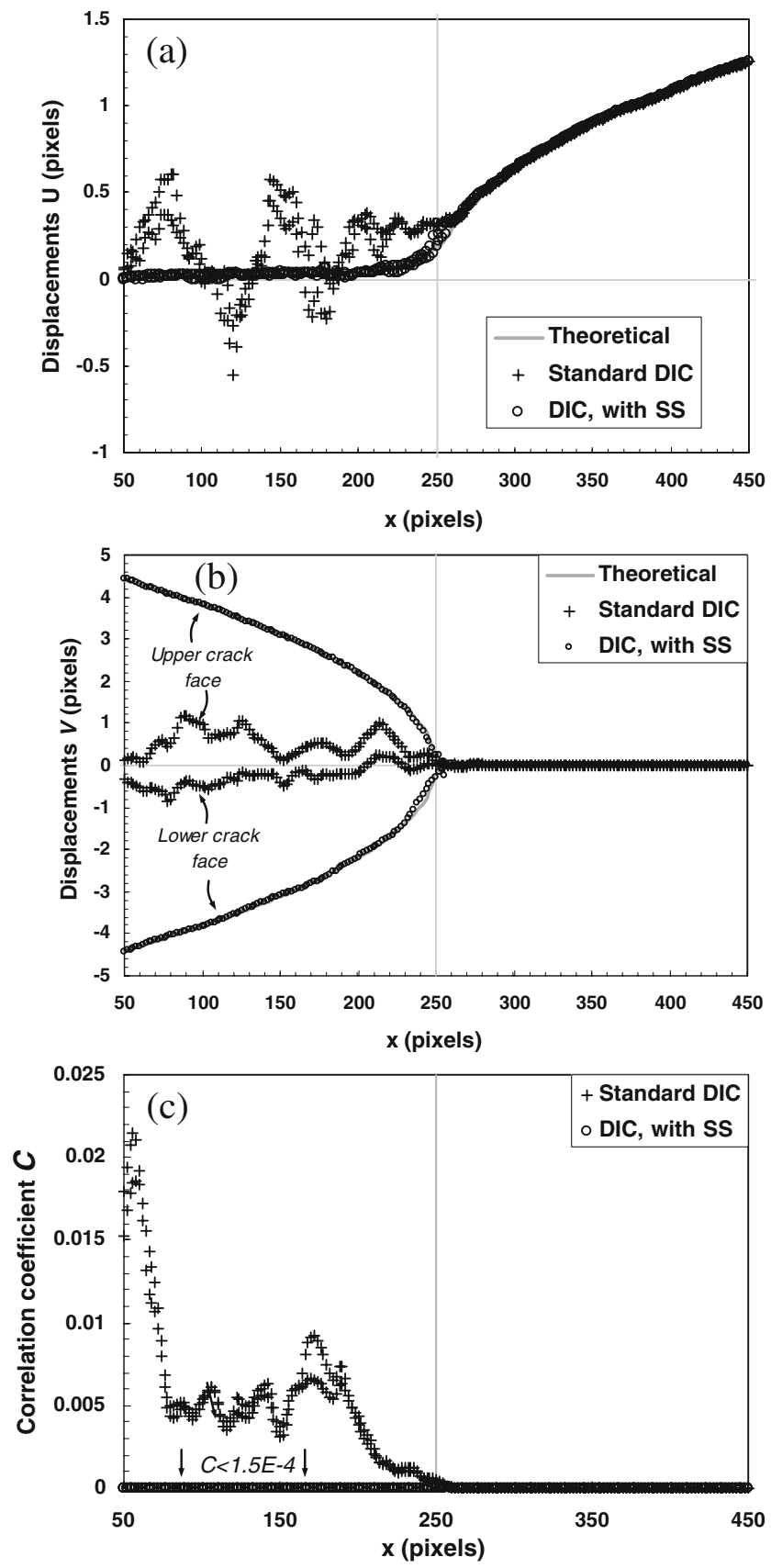

Fig. 6 Data collected along the crack line at \pm 2 pixels from the crack, super-pixel opening in mode I: (a) Displacement $U$, (b) Displacement $V$, (c) Correlation coefficient $C$
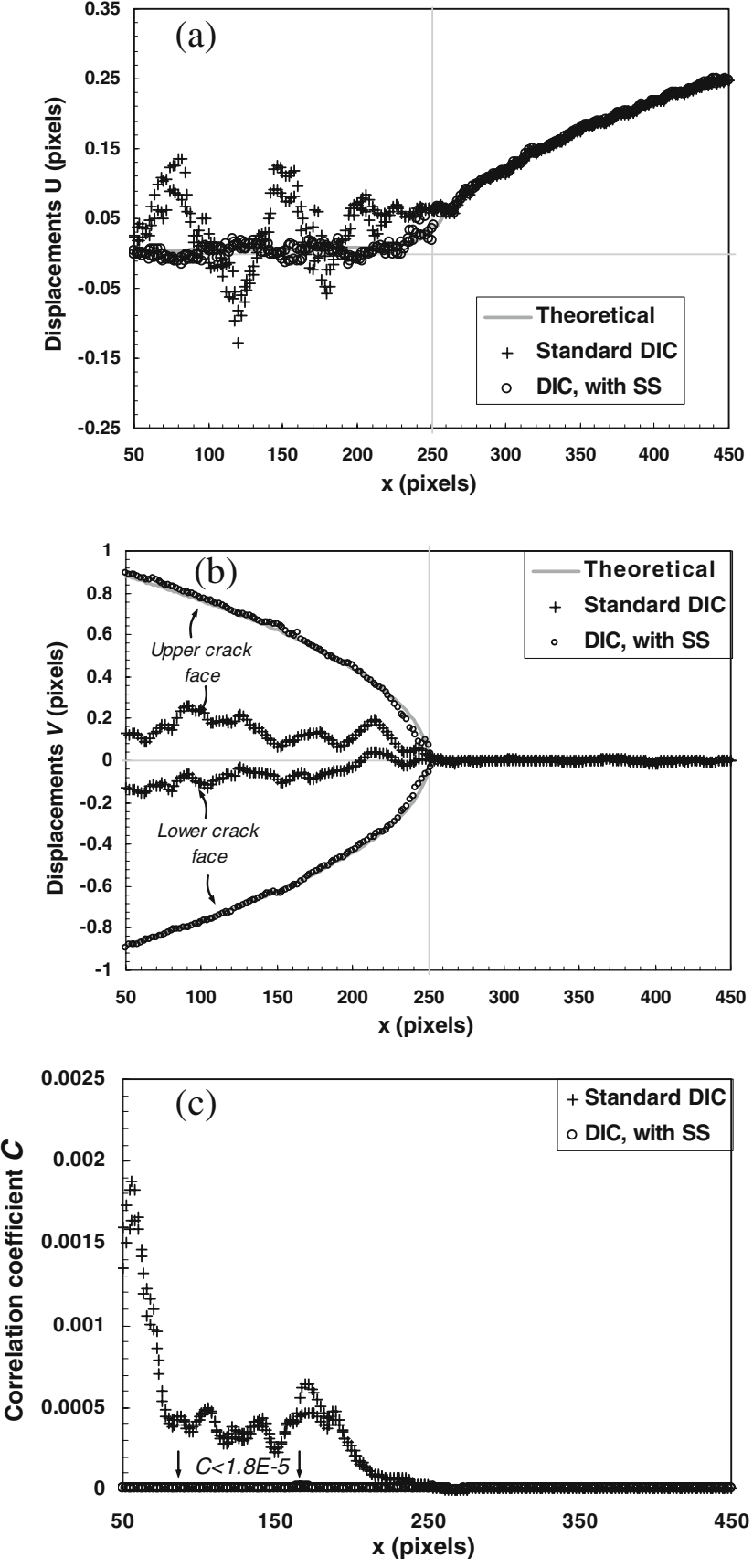

Fig. 7 Data collected along the crack line at \pm 2 pixels from the crack, sub-pixel opening in mode I: (a) Displacement $U$, (b) Displacement $V$, (c) Correlation coefficient $C$

threshold ensured that only the subsets near the crack would undergo subset splitting. Depending on the quality of the images, the splitting threshold must first be adjusted by proper inspection of the correlation coefficients or by trial and error.

The results in Fig. 5 clearly show that standard DIC is unable to capture the displacement jump associated with the apparition of a crack in the image. The vertical displacements seem to vary progressively across the crack and the correlation coefficient is significantly degraded in a band 26 
Fig. 8 Images used for the evaluation of the subset splitting method with a mode II crack at a $35^{\circ}$ angle: (a) Reference image and area of interest; (b) Super-pixel crack sliding; (c) Sub-pixel crack sliding

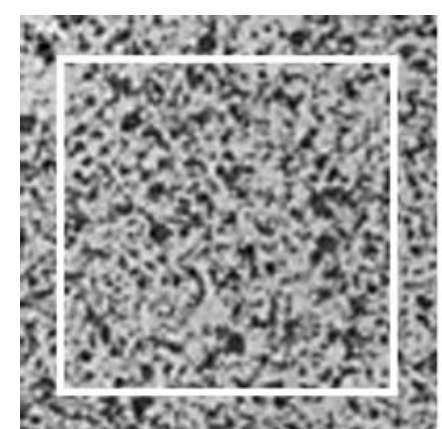

(a)

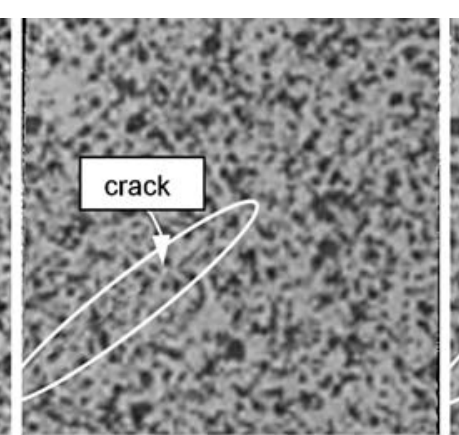

(b)

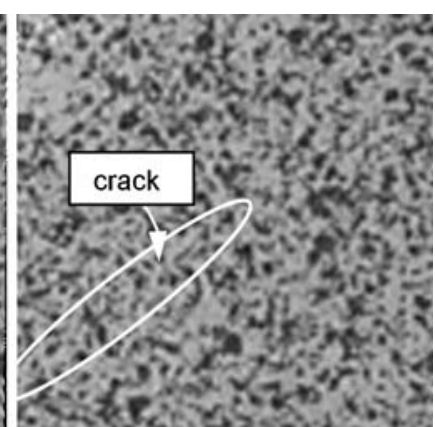

(c)

Fig. 9 Image correlation results from the mode II angled crack with super-pixel displacements using standard DIC and DIC with subset splitting (all axes' units in pixels)

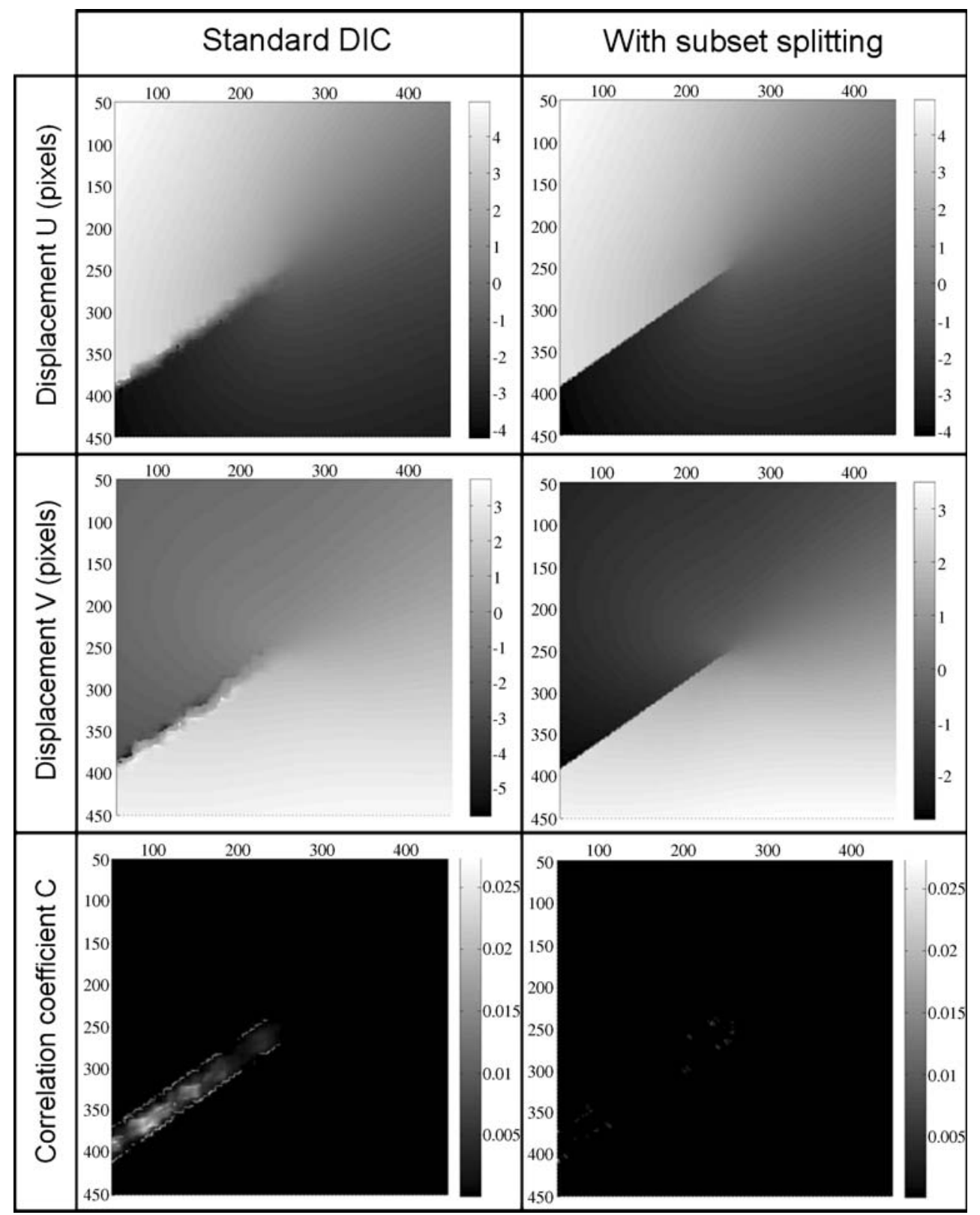


pixels wide (about the width of the subset) centered on the crack (the crack perturbs the correlation as soon as it enters a subset). DIC with subset splitting, on the other hand, properly captures the displacement jump and achieves a better correlation quality over the entire image. Note that the displacement fields from the sub-pixel crack opening images (not shown here) looked similar but with smaller magnitudes of displacements. Figures 6 and 7 show the horizontal displacement $U$, the vertical displacement $V$, and
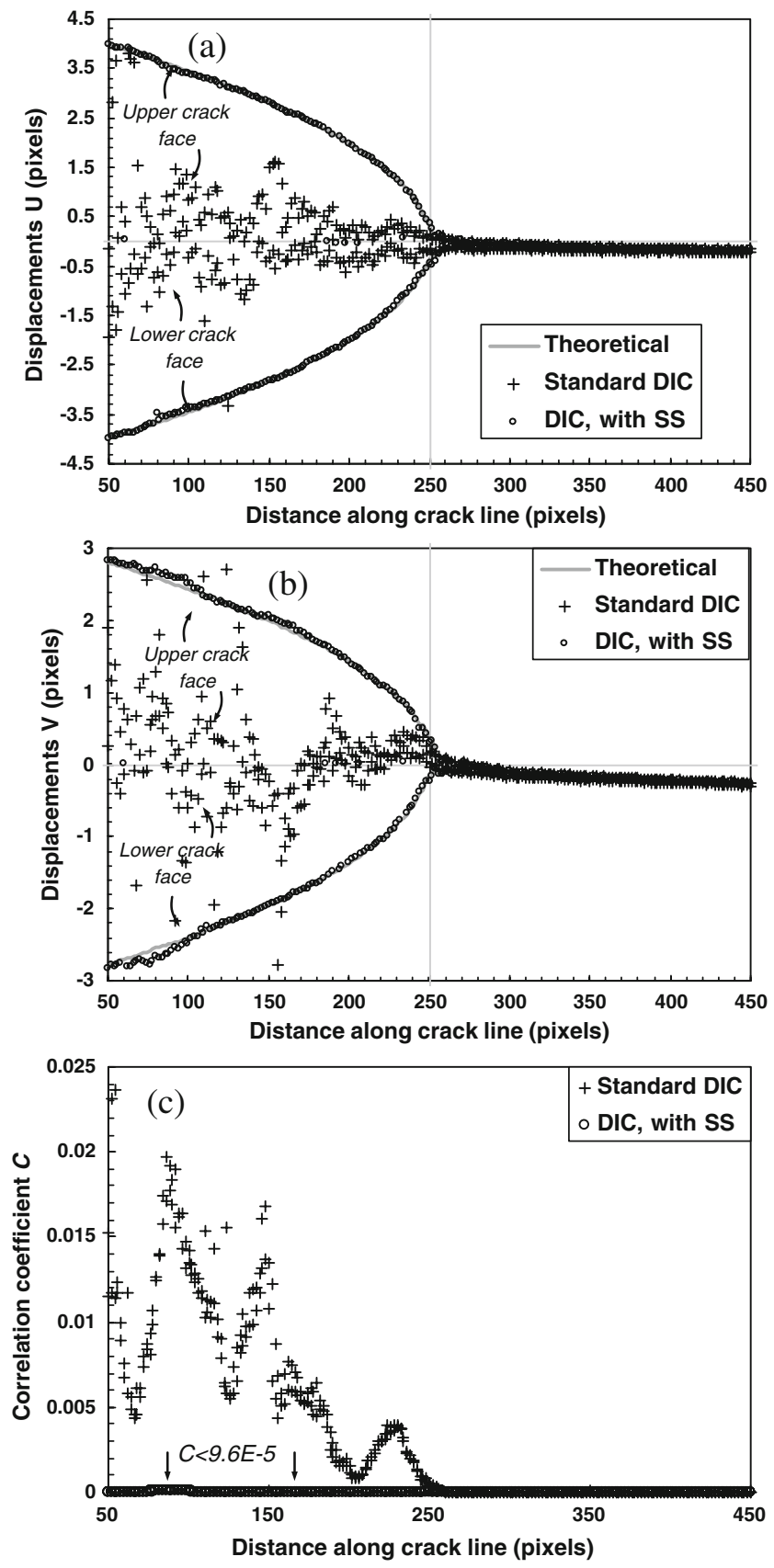

Fig. 10 Data collected along the crack line at \pm 2 pixels from the crack, super-pixel opening in mode II: (a) Displacement $U$, (b) Displacement $V$, (c) Correlation coefficient $C$
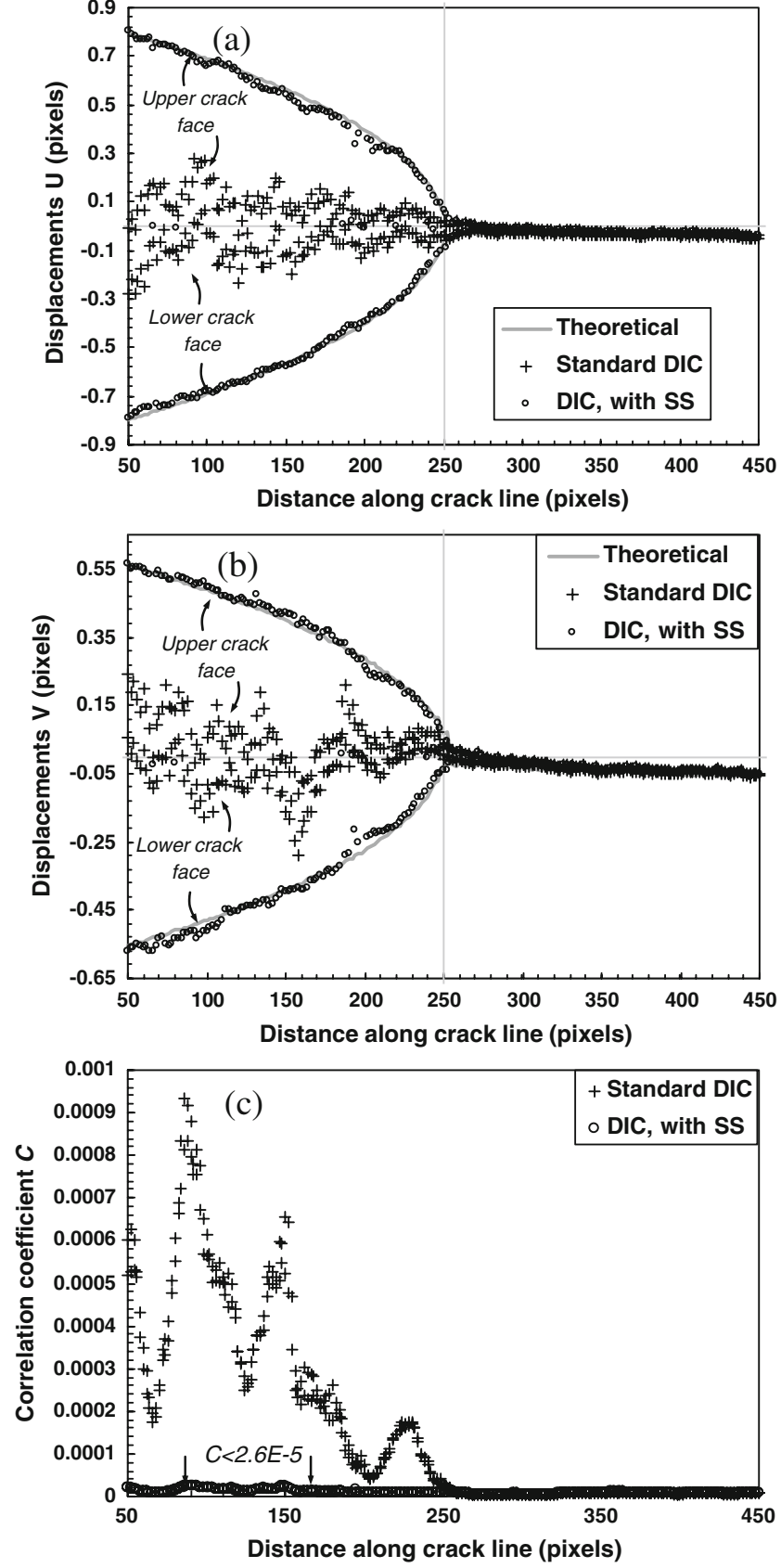

Fig. 11 Data collected along the crack line at \pm 2 pixels from the crack, sub-pixel opening in mode II: (a) Displacement $U$, (b) Displacement $V$, (c) Correlation coefficient $C$

the correlation coefficient $C$ plotted along the crack line at $y= \pm 2$ pixels (i.e. lower and upper crack faces) for the case with super-pixel crack openings (Fig. 6) and sub-pixel crack openings (Fig. 7). Clearly, standard DIC is unable to properly capture the crack profile, returning a poor correlation quality (an average of $C=0.005$ along the crack faces for super-pixel displacements and $C=0.0004$ for subpixel displacements). On the other hand DIC with subset splitting shows a near perfect match with the theoretical displacement field: the average relative error on the 
displacement $V$ along the crack face was $0.54 \%$ for superpixel and $1.30 \%$ for sub-pixel opening displacements, and an average $C=1.45 \mathrm{E}-5$ (super) and $\mathrm{C}=1.06 \mathrm{E}-5$ (sub) was obtained. The error was the largest near the crack tip (ranging from $3 \%$ to $70 \%$ ), because the subset splitting method is currently unable to capture discontinuities which end within the subset. Nevertheless, the correlation quality and the overall result for the subset splitting analysis are far superior to standard DIC near the crack faces.

Example 2: Digitally Created Mode II Crack at an Angle

The subset splitting method was also validated for cases where the discontinuity crosses the subset at an angle and when the displacement jump is tangential to the discontinuity. These two attributes are embodied in this second example; a pure Mode II crack which is located at $35^{\circ}$ from the $X$ axis of the image. The mode II crack displacement field is given by:

$$
\begin{aligned}
U(x, y)= & \frac{K_{\mathrm{II}}}{2 E} \sqrt{\frac{r}{2 \pi}}(1+v)[(2 \kappa+1) \sin (\theta / 2) \\
& -\sin (3 \theta / 2)] \\
V(x, y)= & \frac{K_{\mathrm{II}}}{2 E} \sqrt{\frac{r}{2 \pi}}(1+v)[(2 \kappa-1) \cos (\theta / 2) \\
& -\cos (3 \theta / 2)]
\end{aligned}
$$

Where $(x, y)$ and $(r, \theta)$ are the Cartesian and polar coordinates centered on the crack tip, and aligned with the crack line. $K_{\mathrm{II}}$ is the stress intensity factor. The parameters above were chosen exactly as in example 1 in order to achieve a set of super-pixel crack sliding displacements and a set of sub-pixel crack sliding displacements. The axes and displacements were then rotated by $35^{\circ}$ to obtain the deformed images shown on Figs. 8(b) and (c). The same DIC parameters used in example 1 (subset size, grid size, subset splitting threshold) were used for this set of images. The image correlation results from these images are shown on Fig. 9. As in the first example, the large errors and poor correlation quality returned by standard DIC are significantly improved by using the subset splitting method (subpixel crack sliding images looked similar and are not presented here). Figures 10 and 11 show the displacements $U$ and $V$, and the correlation coefficient along the crack line. Theoretical displacements and displacements from DIC were again in good agreement when subset splitting was used. Along the crack the average relative error on the magnitude of the displacements was found to be $0.64 \%$ for super-pixel displacements and $2.18 \%$ for sub-pixel displacements. Subset splitting can therefore effectively capture the tangential displacement jump along the angled line. Another potential application could therefore be the study of shear bands, which produce similar displacement discontinuities to those from this example.

Example 3: Actual Fracture Experiment on Single Edge Notch Bend (SENB) High Density Polyethylene (HDPE) Specimen

The third example was chosen to demonstrate the robustness of the subset splitting method with actual experimental
Fig. 12 Images taken during the fracture experiment. (a) Reference image with correlation area of interest; (b), (c), (d) Three deformed images taken as three different crack extensions

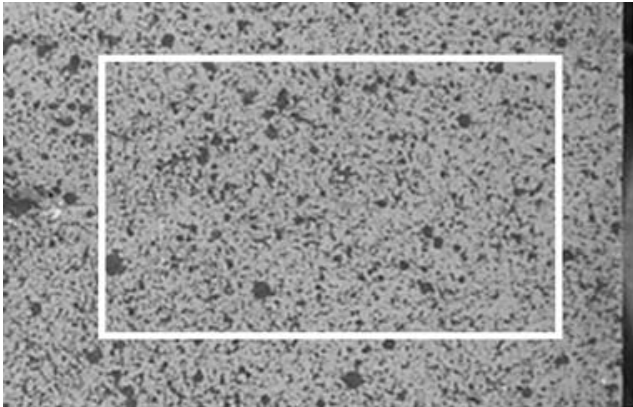

(a)

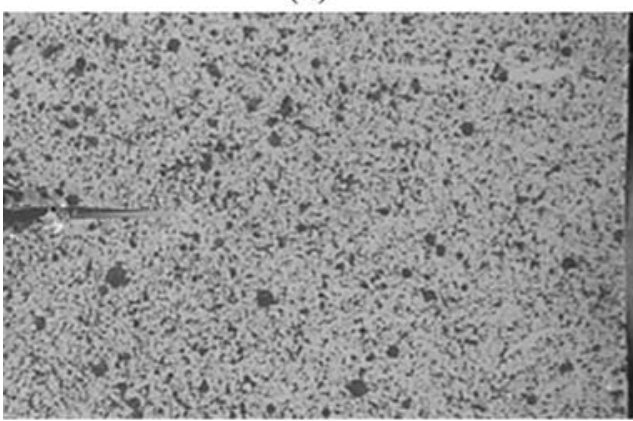

(c)

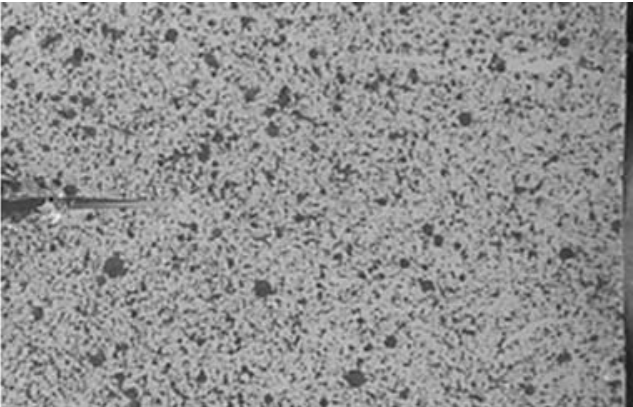

(b)

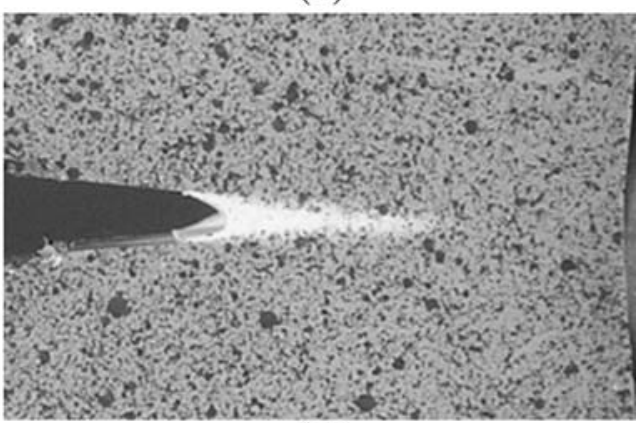

(d) 
Fig. 13 DIC results for three pairs of images from the actual SENB experiment where the crack is advancing: (a) Vertical displacement and (b) Vertical strain (axes' units in millimeters)
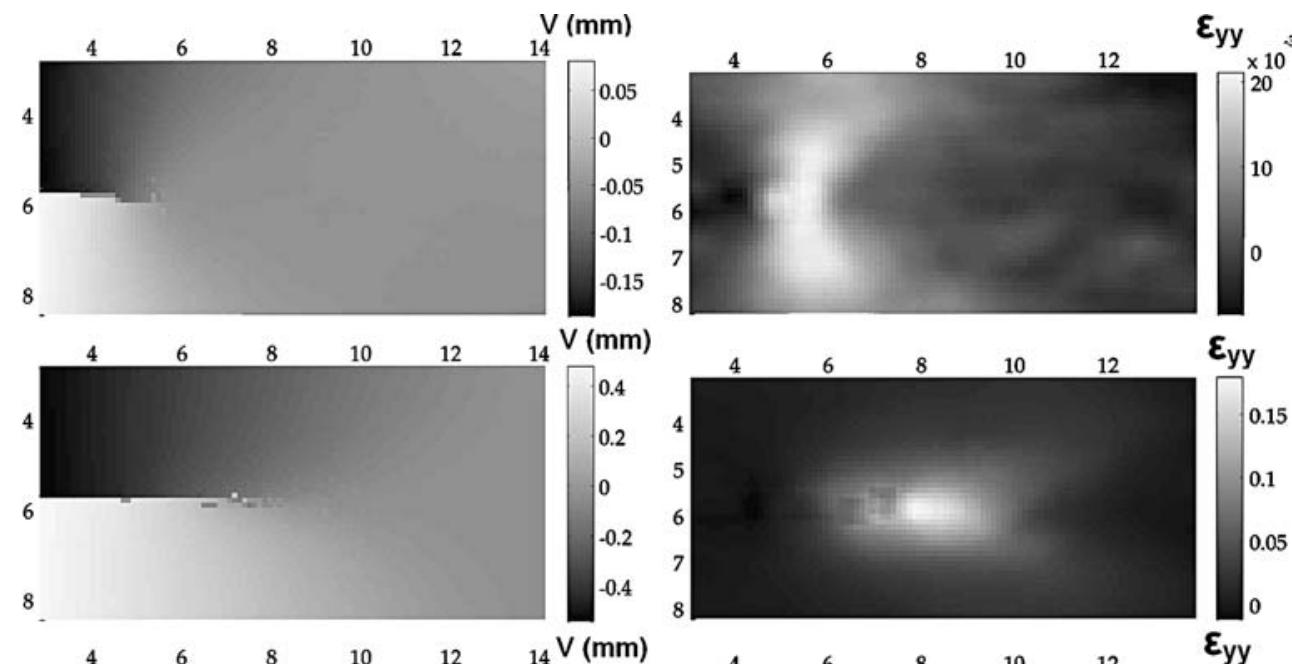

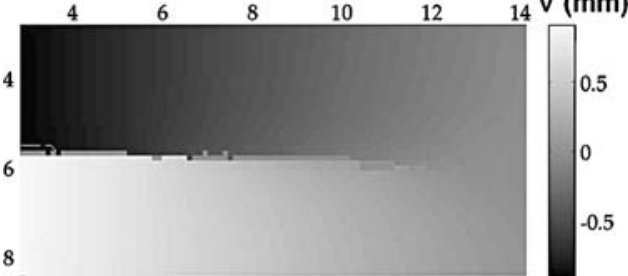

(a)

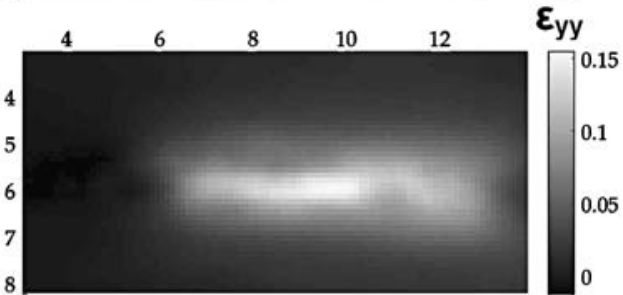

(b) images. A High Density Polyethylene (HDPE) specimen was tested in Single Edge Notched Bending configuration (SENB) following ASTM standard D5045-99 [20]. HDPE was chosen for its ductility and toughness - a stable crack can easily be propagated and imaged in this material. The specimen was cut to a length of $114.3 \mathrm{~mm}$, a width of $25.4 \mathrm{~mm}$, and a thickness of $12.7 \mathrm{~mm}$. The initial notch was generated first with a band saw cut, and then by tapping a fresh razor blade so that the final notch was approximately half the width of the specimen in length [20]. In order to generate a speckle pattern on the surface, small mists of black spray paint were applied by hand. The surface of HDPE being naturally opaque and white; a good quality speckle pattern was easily obtained with black spray paint. The specimen was loaded in three-point bending on a universal loading machine (MTS systems corporation, Eden Prairie, MN, USA). During the experiment about forty $1,600 \times 1,200$ TIFF images of the sample surface were captured at regular 8 second intervals using a digital camera on a tripod (Olympus C5060, Olympus America Center Valley, PA, USA). Figure 12 shows a sequence of images used for correlation. The reference image was taken from the unloaded specimen, and the deformed images show the specimen before and after crack propagation. Note that for this material, white ligaments across the crack can be seen on a short distance behind the crack tip. The subset size was chosen as 25 and the grid size as 2 pixels. The splitting threshold was chosen as 0.004 for the early images, but moved up to 0.01 for larger deformations. Figure 13(a) shows that the subset splitting method could properly capture the crack propagation and the associated displacement jump, and that the displacements could be determined very close to the crack faces. Figure 14 shows the crack opening displacement (i.e. the profile of the crack) for different crack lengths. The method is robust and captures the displacement jumps along the entire crack. The next step was the calculation of the strains, which is usually done by finite differences and low pass filtering of the displacements (or any other equivalent technique). In this case this method yields results near the discontinuity which are not representative of the actual strains, because of the perturbation from the displacement jump. The strains near

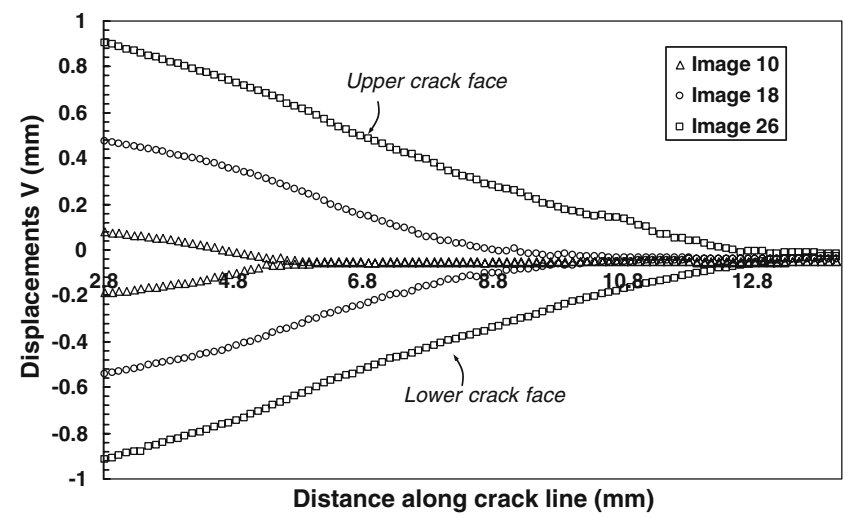

Fig. 14 Crack profiles for the three images (Displacements $V$ were collected along two horizontal lines right above and below the crack line) 
the discontinuity were therefore directly taken from the deformation of the master section for the individual subsets. The resulting strain maps for $\varepsilon_{y y}$ are shown in Fig. 13(b). The subset splitting method captured the strains in the wake of the crack, including near the crack faces. This type of capability could be used to compute $J$ integrals more accurately than with the standard method alone. In addition, the results could capture the shape of the inelastic region ahead of the crack tip, which can be relevant in modeling certain classes of toughening mechanisms associated with phase transformation.

\section{Conclusions}

Digital image correlation is now a well-established experimental mechanics technique for measuring displacements and strains on a material surface. However a significant limitation of the basic technique is poor results near discontinuities. Here an improved approach to DIC was introduced to overcome this limitation, where the subset is essentially split into two sections along a line, and where each of these sections is matched using independent deformation parameters. To implement this approach, an algorithm was presented and was validated using two digitally deformed images: one for a horizontal crack in Mode I, and the other for a Mode II crack at an angle. The validation showed that the subset splitting method can accurately capture sub-pixel and super-pixel displacement jumps across discontinuities. Image correlations from an actual fracture test were also presented to demonstrate the robustness of the method on real images. This subset splitting DIC method therefore provides a robust and accurate method to capture displacement jumps and strains near discontinuities, which is a clear improvement over standard DIC. For example crack opening profiles, shapes and sizes of inelastic regions or residual strains in the wake are now accessible through image correlation, which make this approach attractive in experimental fracture mechanics. Compared to other methods that can handle discontinuities, subset splitting DIC offers the advantage of being a direct extension of the now popular standard DIC technique. As such, this new technique can be implemented as an "add-on" by researchers who already use standard DIC. The method can also probably be adapted to handle three-dimensional discontinuities on the basis of digital volume correlation.

Acknowledgements This work was supported by the McGill University Faculty of Engineering. Jeffrey Poissant was supported by an Undergraduate Student Research Award from the Natural Sciences and Engineering Research Council of Canada during the summer of 2007. The authors would like to thank Jonathan Laliberté for his assistance on the experiments.

\section{References}

1. Sutton MA, Wolters WJ, Peters WH, Ranson WF, McNeill SR (1983) Determination of displacements using an improved digital correlation method. Image Vis Comput 13:33-139 doi:10.1016/ 0262-8856(83)90064-1

2. Chu TC, Ranson WF, Sutton MA, Peters WH (1985) Applications of digital-image-correlation techniques to experimental mechanics. Exp Mech 253:232-244 doi:10.1007/BF02325092

3. McNeill SR, Peters WH, Sutton MA (1987) Estimation of stress intensity factor by digital image correlation. Eng Fract Mech 281:101-112 doi:10.1016/0013-7944(87)90124-X

4. Chasiotis I, Cho SW, Jonnalagadda K (2006) Fracture toughness and subcritical crack growth in polycrystalline silicon. ASME 73:714-722

5. Rechenmacher AL (2006) Grain-scale processes governing shear band initiation and evolution in sands. J Mech Phys Solids 541:22-45 doi:10.1016/j.jmps.2005.08.009

6. Sutton MA, Turner JL, Chao YJ, Bruck HA, Chae TL (1992) Experimental investigations of three-dimensional effects near a crack tip using computer vision. Int J Fract 53(3):201-228

7. Gonzalez J, Knauss WG (1998) Strain inhomogeneity and discontinuous crack growth in a particulate composite. J Mech Phys Solids 4610:1981-1995 doi:10.1016/S0022-5096(98)00037-4

8. Chao YJ, Luo PF, Kalthoff JF (1998) An experimental study of the deformation fields around a propagating crack tip. Exp Mech 382:79-85 doi:10.1007/BF02321648

9. Barthelat F, Espinosa HD (2007) An experimental investigation of deformation and fracture of nacre-mother of pearl. Exp Mech 473:311-324 doi:10.1007/s11340-007-9040-1

10. Helm J (2008) Digital image correlation for specimens with multiple growing cracks. Exp Mech 48:753-762 doi:10.1007/ s11340-007-9120-2

11. Jin H, Bruck HA (2005) Pointwise digital image correlation using genetic algorithms. Exp Tech 291:36-39 doi:10.1111/j.1747-1567. 2005.tb00202.x

12. Réthoré J, Hild F, Roux S (2008) Extended digital image correlation with crack shape optimization. Int J Numer Methods Eng 732:248-272 doi:10.1002/nme.2070

13. Réthoré J, Hild F, Roux S (2007) Shear-band capturing using a multiscale extended digital image correlation technique. Comput Methods Appl Mech Eng 196(49-52):5016-5030 doi:10.1016/j. cma.2007.06.019

14. Moes N, Belytschko T (2002) Extended finite element method for cohesive crack growth. Eng Fract Mech 697:813-833 doi:10.1016/ S0013-7944(01)00128-X

15. Bruck HA, McNeill SR, Sutton MA, Peters WH (1989) Digital image correlation using Newton-Raphson method of partialdifferential correction. Exp Mech 293:261-267 doi:10.1007/ BF02321405

16. Vendroux G, Knauss WG (1998) Submicron deformation field measurements: Part 2. Improved digital image correlation. Exp Mech 382:86-92 doi:10.1007/BF02321649

17. Schreier HW, Braash JR, Sutton MA (2000) Systematic errors in digital image correlation caused by intensity interpolation. Exp Mech 39(11):2915-2921

18. Schreier H, Sutton M (2002) Systematic errors in digital image correlation due to undermatched subset shape functions. Exp Mech 423:303-310 doi:10.1007/BF02410987

19. Pan B, Xie H, Wang Z, Qian K, Wang Z (2008) Study on subset size selection in digital image correlation for speckle patterns. Opt Express 1610:7037-7048 doi:10.1364/OE.16.007037

20. D5045-99 AS (1999) Standard test methods for plane-strain fracture toughness and strain energy release rate of plastic materials. ASTM International 